\title{
Efficacy and Safety of Globin Digest as a Functional Beverage for the Treatment of Childhood Obesity
}

\author{
Daisuke Tokuhara ${ }^{1, *}$, Eri Hayashi ${ }^{2}$, Yuki Cho ${ }^{1}$, Haruo Shintaku ${ }^{1}$ \\ ${ }^{1}$ Department of Pediatrics, Osaka City University Graduate School of Medicine, Osaka, Japan \\ ${ }^{2}$ Department of Pediatrics, Izumi Municipal Hospital, Osaka, Japan \\ *Corresponding author: m1155519@med.osaka-cu.ac.jp
}

\begin{abstract}
Background and aims: Childhood obesity is a serious health concern in many countries in the world. In addition to lifestyle education, functional foods or beverages may be useful for preventing and treating obesity. Here we evaluated the effectiveness and safety of a globin digest (GD) functional beverage for the treatment of childhood obesity. Methods: Twenty-eight obese Japanese children (median age, 12.4 years; range, 6.0-17.3 years; 18 males) were randomly assigned to receive orally either placebo beverage $(n=14)$ or GD beverage containing 6 mg of the amino acid residue Val-Val-Tyr-Pro $(n=14)$ within $1 \mathrm{~h}$ of eating dinner daily for 3 months. Changes in body weight, body mass index percentile, and percentage overweight were based on Japanese age-, sex-, and heightspecific body-weight standards. The prevalence of adverse events was also evaluated. Results: The changes in body weight, body mass index percentile, or percentage overweight were not significantly different between the two groups at 3 months or at 6 months after the start of GD intake. No adverse events were reported in any of the subjects. Conclusions: Although the effectiveness of GD beverages for the treatment of childhood obesity needs further evaluation, the GD beverage used in the present study was safe and had good compliance in obese Japanese children.
\end{abstract}

\section{Keywords: globin digest, obesity, children, triglyceride, functional beverage}

Cite This Article: Daisuke Tokuhara, Eri Hayashi, Yuki Cho, and Haruo Shintaku, "Efficacy and Safety of Globin Digest as a Functional Beverage for the Treatment of Childhood Obesity." Journal of Food and Nutrition Research, vol. 5, no. 4 (2017): 281-284. doi: 10.12691/jfnr-5-4-10.

\section{Introduction}

Childhood obesity is a serious health concern in many countries in the world [1,3,4]. In developed countries, between $20 \%$ and $25 \%$ of children are overweight or obese [1]. In addition, childhood obesity is a leading cause of childhood chronic liver disease, and $22.5 \%$ to $38 \%$ of obese children have fatty liver compared with $2.6 \%$ to $9.6 \%$ of the general pediatric population [5,6]. Obese children are likely to remain obese into adulthood [2], and therefore will be at increased risk of developing non-alcoholic fatty liver disease, hypertension, hyperlipidemia, and diabetes mellitus [16,17,18,19]. Lifestyle education during primary care visits to emphasize the need for an appropriate diet and exercise routine is an important basic strategy to treat childhood obesity $[7,8,9,10]$; however, the success of these efforts is often hampered by the limited contact the child has with their primary care facility $[8,9,10]$. Thus, there is interest in using drugs or functional foods and beverages that are easily taken at home and could help prevent or reduce childhood obesity $[11,12,13,21,22,23]$.

Obesity occurs most often as a consequence of excessive caloric intake resulting in increased storage of triglycerides in adipose tissue [20]; therefore, pharmacological inhibition of triglyceride (TG) synthesis or absorption is a potential therapeutic strategy to reduce obesity [11,12,13,22,23]. Previously, globin digest (GD), an oligopeptide mixture derived from edible globin proteins, was developed as a functional food or beverage and has been shown to be effective at reducing hyperlipidemia and to have hepatoprotective effects in humans and other mammals $[12,13,14]$. The amino acid residue contained in GD, Val-Val-Tyr-Pro, inhibits the absorption of fat in the digestive tract and enhances the activity of hepatic triglyceride lipase in mice [12]. Furthermore, a clinical study has shown that postprandial serum TG and chylomicron levels are suppressed after intake of GD in healthy individuals [13]. Although GD is a promising means of reducing obesity, the efficacy and long-term safety of GD in both children and adults with obesity remains unknown. Here we evaluated the efficacy and safety of GD in obese Japanese children.

\section{Materials and Methods}

\subsection{Materials}

The GD (MG Pharma Incorporation, Osaka, Japan) used in the present study was produced from the enzymatic hydrolysis of globin protein in bovine or swine hemoglobin, as previously described [13]. The GD was manufactured as a $50-\mathrm{mL}$ beverage containing $6 \mathrm{mg}$ of the 
amino acid residue Val-Val-Tyr-Pro. A placebo beverage with a similar taste and appearance was produced at the same factory that produced the GD beverage.

\subsection{Patients}

Twenty-eight Japanese obese children (median, 12.4 years; range, 6.0-17.3 years; 18 males) were enrolled in the study between November 2012 and April 2013. For each child, percentage overweight (POW) based on the Japanese age-, sex-, and height-specific bodyweight standards was calculated and obesity was diagnosed if POW was greater than or equal to $20 \%$, which is the definition that has been adopted by the Ministry of Health, Labor and Welfare of Japan [15]. Because body fat percentage is not required for the diagnosis of obesity [15], we used POW ( $\geq 20 \%)$ for the inclusion criteria which is modified after trial commencement. Children without obesity, or with chronic intestinal diseases associated with absorption or digestion failure, severe liver disorder, kidney disease, other known severe underlying disease or past history of severe illness, or females who are pregnant, breastfeeding or intended to become pregnant during study were excluded. To analyze the baseline biochemical characteristics of the subjects, blood was drawn before the start of GD intake and serum concentrations of aspartate aminotransferase (AST), alanine aminotransferase (ALT), and TG were determined by means of standard laboratory blood tests. Serum concentrations of total cholesterol, high-density lipoprotein, low-density lipoprotein, glucose and insulin were not evaluated in some of participants because of lack of enough amount of blood sample thus excluded from the baseline characteristics determination after trial commencement.

\subsection{Study Design}

This study was a randomized, double-blind, placebocontrolled trial conducted at Osaka City University Hospital. Participants are randomly allocated to one of the two study groups (GD or placebo) by using dynamic allocation that minimizes the differences between groups as participants enter the study. Balancing factors were sex, age, and body weight. The trial conformed to the Helsinki Declaration and was approved by the ethical committees of Osaka City University Graduate School of Medicine (the approved number, 2394; UMIN trial ID, UMIN000021826). Written informed consent was obtained from each child' s parent or legal guardian, and written assent was obtained from children who were at least 6 years old, after children and each child' s parent or legal guardian were given, in writing, a full explanation of the aims of the study, its possible hazards, discomfort, and inconvenience.

\subsection{Interventions}

Fourteen subjects were randomly assigned to orally receive GD beverage, and 14 subjects were randomly assigned to receive placebo beverage within $1 \mathrm{~h}$ of dinner daily for 3 months. Children under 12 years were given 25 $\mathrm{mL}$ of placebo or GD beverage; children 12 to 18 years old were given $50 \mathrm{~mL}$ of placebo or GD beverage. It was difficult for patients and legal guardians to have regular medical visits every 4 weeks during research period described in the approved study protocol, thus patients were evaluated after 3 and 6 months of GD or placebo intake. Patients were blinded, and all clinical assessments were performed without knowledge of the assigned treatment. In both groups, brief lifestyle counseling was provided.

\subsection{Outcomes}

Changes in body weight, POW, and body mass index (BMI) percentile for age were compared between the groups at 3 and 6 months after study initiation. Patients were asked to report adverse events such as vomiting, diarrhea, abdominal pain, or skin eruption.

\subsection{Statistical Analysis}

Comparisons of patient baseline characteristics and outcomes between the GD group and the placebo group were performed by using the Mann-Whitney $U$ test. $P$ values $<0.05$ were considered statistically significant.

\section{Results}

\subsection{Baseline Clinical and Biochemical Characteristics}

There were no significant differences in the baseline characteristics of sex, age, height, body weight, POW, and BMI percentile between the GD and placebo groups (Table 1). Serum levels of TG, AST, and ALT at the start of the study were also not significantly different between the two groups.

Table 1. Baseline patient demographics and biochemical parameters

\begin{tabular}{cccc}
\hline & GD & Placebo & P value \\
\hline $\mathrm{n}$ & 14 & 14 & \\
Male & 8 & 10 & NS \\
Age (years) & $12.4 \pm 2.8$ & $12.6 \pm 2.8$ & NS \\
Height (cm) & $154.9 \pm 16.5$ & $151.5 \pm 14.4$ & NS \\
BW (kg) & $72.9 \pm 31.9$ & $71.2 \pm 27.3$ & NS \\
POW (\%) & $53.2 \pm 33.6$ & $57.4 \pm 31.2$ & NS \\
BMI percentile & $97.3 \pm 2.2$ & $97.4 \pm 2.7$ & $N S$ \\
TG (mg/dL) & $99.2 \pm 39.0$ & $134.4 \pm 77.0$ & NS \\
AST (U/L) & $58.3 \pm 78.9$ & $44.9 \pm 67.7$ & NS \\
ALT (U/L) & $85.6 \pm 119.1$ & $62.9 \pm 108.5$ & NS \\
\hline
\end{tabular}

GD, globin digest; BW, body weight; POW, percentage overweight based on Japanese age-, sex-, and height-specific body-weight standards; BMI, body mass index; TG, triglyceride; AST, aspartate aminotransferase; ALT, alanine aminotransferase; NS, not significant.

\subsection{Adverse Events}

All of the subjects finished the 3-month course of daily oral placebo or GD beverage. During the trial period, no adverse events related to GD intake were reported. 


\subsection{Effects of GD on Obesity}

There were no significant differences in change in body weight, BMI percentile, or POW between the two groups at 3 months and at 6 months after the start of GD intake (Table 2). Furthermore, when the numbers of patients with a reduction in body weight, POW, or BMI percentile were compared between the groups, there were no significant differences: body weight (8 in GD group vs. 8 in placebo group), POW (6 vs. 5), and BMI percentile (5 vs. 4), suggesting that GD may induce a placebo effect that results in weight loss.

Table 2. Outcome of globin digest (GD) beverage intake

\begin{tabular}{lccc}
\hline & GD & Placebo & $P$ value \\
\hline Change in BW (kg) & & & \\
At month 3 & $0.0 \pm 2.9$ & $-0.6 \pm 2.2$ & NS \\
At month 6 & $1.6 \pm 4.0$ & $0.7 \pm 4.1$ & NS \\
Change in POW (\%) & & & \\
At month 3 & $1.6 \pm 6.7$ & $0.7 \pm 5.0$ & NS \\
At month 6 & $0.1 \pm 8.1$ & $-2.8 \pm 10.3$ & NS \\
Change in BMI percentile & & & \\
At month 3 & $1.0 \pm 3.8$ & $0.2 \pm 1.0$ & NS \\
At month 6 & $-0.6 \pm 2.8$ & $-0.5 \pm 2.0$ & NS \\
\hline
\end{tabular}

BW, body weight; POW, percentage overweight based on Japanese age-, sex-, and height-specific body-weight standards; BMI, body mass index; NS, not significant.

Table 3. Number of patients who showed a reduction in body weight (BW), percent overweight (POW), or body mass index (BMI) percentile at 3 months after the end of treatment

\begin{tabular}{lccc}
\hline & $\begin{array}{c}\text { GD } \\
(\mathrm{n}=14)\end{array}$ & $\begin{array}{c}\text { Placebo } \\
(\mathrm{n}=14)\end{array}$ & P value \\
\hline BW (kg) & 8 & 8 & $N S$ \\
POW (\%) ${ }^{1}$ & 6 & 5 & $N S$ \\
BMI percentile & 5 & 4 & $N S$ \\
\hline
\end{tabular}

GD, globin digest; NS, not significant.

${ }^{1}$ POW was calculated based on Japanese age-, sex-, and height-specific body-weight standards

\section{Discussion}

Functional foods and beverages may be useful to treat obesity in children. Here we found that a GD beverage did not produce any side effects and was safe and had good compliance in the obese Japanese pediatric population. However, we found that the GD beverage did not have a significant effect on the body weight between the GD and the placebo group.

The present study had several limitations. The first was that it was difficult to standardize fat intake among the subjects. A previous study demonstrated that GD effectively reduced hyperlipidemia in healthy adults that were ingesting standardized amounts of fat and calories [13]. However, in the present study it was difficult to standardize fat intake because the age, gender, and lifestyle, including favorite foods, varied widely among the children. Therefore, in future studies, it may be necessary to compare the fat and caloric intake between the two groups based on diet records.
The second limitation was that there have been no previous studies demonstrating the effects of GD in obese children, so it was not easy to determine suitable dosages and timing of GD intake. Because obese children ingest excessive amounts of calories or fat, or both, at each meal, and GD is expected to reduce the postprandial absorption of fat, it may be necessary to give the GD beverage not once daily but three times daily after each meal. The third limitation concerns the lifestyle education the children received. Encouragement was provided to the children to improve their lifestyle at each medical visit; therefore, this lifestyle education may have contributed to the suspected placebo effect of the GD beverage.

In conclusion, although we did not show a difference between the GD and placebo groups in the current study design, the limitations of the study mean that we are unable to conclude that the GD beverage had no effect on the body weight of the subjects. Since we did show that the GD beverage used in the present study was safe and had good compliance in obese Japanese children, we recommend that a large group study be conducted to further clarify whether or not GD beverages are effective for reducing obesity in the pediatric population.

\section{Acknowledgements}

The globin digest and placebo beverages used in this study were provided by MG Pharma Incorporation (Osaka, Japan). The authors have no other competing interests.

\section{Abbreviations}

TG, triglyceride; GD, globin digest; POW, percentage overweight; AST, aspartate aminotransferase; ALT, alanine aminotransferase; BMI, body mass index; BW, body weight

\section{References}

[1] Ng, M., Fleming, T., Robinson, M., Thomson, B., Graetz, N., Margono, C., Mullany, E.C., Biryukov, S., Abbafati, C., et al, "Global, regional, and national prevalence of overweight and obesity in children and adults during 1980-2013: a systematic analysis for the Global Burden of Disease Study 2013," Lancet, 384 (9945). 766-781. Aug 2014.

[2] Togashi, K., Masuda, H., Rankinen, T., Tanaka, S., Bouchard, C. and Kamiya, H, "A 12-year follow-up study of treated obese children in Japan," Int J Obes Relat Metab Disord, 26 (6). 770-777. Jun 2002.

[3] Ogden, C.L., Carroll, M.D., Kit, B.K. and Flegal, K.M, "Prevalence of childhood and adult obesity in the United States, 2011-2012," JAMA, 311 (8). 806-814. Feb 2014.

[4] Matsushita, Y., Yoshiike, N., Kaneda, F., Yoshita, K. and Takimoto, $\mathrm{H}$, "Trends in childhood obesity in Japan over the last 25 years from the national nutrition survey," Obes Res, 12 (2). 205-214. Feb 2004.

[5] Tominaga, K., Kurata, J.H., Chen, Y.K., Fujimoto, E., Miyagawa, S., Abe, I. and Kusano, Y, "Prevalence of fatty liver in Japanese children and relationship to obesity. An epidemiological ultrasonographic survey," Dig Dis Sci, 40 (9). 2002-2009. Sep 1995.

[6] Schwimmer, J.B., Deutsch, R., Kahen, T., Lavine, J.E., Stanley, C. and Behling, C, "Prevalence of fatty liver in children and adolescents," Pediatrics, 118 (4). 1388-1393. Oct 2006. 
[7] Wadden, T.A., Volger, S., Sarwer, D.B., Vetter, M.L., Tsai, A.G., Berkowitz, R.I., Kumanyika, S., et al, "A two-year randomized trial of obesity treatment in primary care practice," $N$ Engl J Med, 365 (21). 1969-1979. Nov 2011.

[8] Martin, P.D., Dutton, G.R., Rhode, P.C., Horswell, R.L., Ryan, D.H. and Brantley, P.J, "Weight loss maintenance following a primary care intervention for low-income minority women," Obesity (Silver Spring), 16 (11). 2462-2467. Nov 2008.

[9] Christian, J.G., Bessesen, D.H., Byers, T.E., Christian, K.K Goldstein, M.G. and Bock, B.C, "Clinic-based support to help overweight patients with type 2 diabetes increase physical activity and lose weight," Arch Intern Med, 168 (2). 141-146. Jan 2008.

[10] Ockene, I.S., Hebert, J.R., Ockene, J.K., Saperia, G.M., Stanek, E., Nicolosi, R., Merriam, P.A. and Hurley, T.G, "Effect of physiciandelivered nutrition counseling training and an office-support program on saturated fat intake, weight, and serum lipid measurements in a hyperlipidemic population: Worcester Area Trial for Counseling in Hyperlipidemia (WATCH)," Arch Intern Med, 159 (7). 725-731. Apr 1999.

[11] Chen, H.C. and Farese, R.V. Jr, "Inhibition of triglyceride synthesis as a treatment strategy for obesity: lessons from DGAT1-deficient mice," Arterioscler Thromb Vasc Biol, 25 (3). 482-486. Mar 2005.

[12] Kagawa, K., Matsutaka, H., Fukuhama, C., Watanabe, Y. and Fujino, H, "Globin digest, acidic protease hydrolysate, inhibits dietary hypertriglyceridemia and Val-Val-Tyr-Pro, one of its constituents, possesses most superior effect," Life Sci, 58 (29). 1745-1755. Apr 1996.

[13] Kagawa, K., Matsutaka, H., Fukuhama, C., Fujino, H. and Okuda, $\mathrm{H}$, "Suppressive effect of globin digest on postprandial hyperlipidemia in male volunteers," J Nutr, 128 (1). 56-60. Jan 1998.

[14] Yamamoto, K., Sasakawa, Y., Nakaoka, F., Nakao, M., Nakamura, M., Kominami, A., Abe, M., Fukuhama, C. and Kagawa K, "Effect of globin digest on the liver injury and hepatic gene expression profile in galactosamine-induced liver injury in SD rats," Life Sci, 88 (15-16). 701-712. Apr 2011.

[15] Asayama, K., Ozeki, T., Sugihara, S., Ito, K., Okada, T., Tamai, H., Takaya, R., Hanaki, K. and Murata M, "Criteria for medical intervention in obese children: a new definition of 'obesity disease' in Japanese children," Pediatr Int, 45 (5). 642-646. Oct 2003.

[16] Lloyd-Jones, D., Adams, R., Carnethon, M., De Simone, G., Ferguson, T.B., Flegal, K., et al. American Heart Association Statistics Committee and Stroke Statistics Subcommittee, "Heart disease and stroke statistics--2009 update: a report from the American Heart Association Statistics Committee and Stroke Statistics Subcommittee," Circulation, 119 (3). 480-486. Jan 2009.

[17] Mokdad, A.H., Ford, E.S., Bowman, B.A., Dietz, W.H., Vinicor, F., Bales, V.S. and Marks, J.S, "Prevalence of obesity, diabetes, and obesity-related health risk factors, 2001," JAMA, 289 (1). 7679. Jan 2003.

[18] Luyckx, F.H., Desaive, C., Thiry, A., Dewé, W., Scheen, A.J., Gielen, J.E. and Lefèbvre, P.J, "Liver abnormalities in severely obese subjects: effect of drastic weight loss after gastroplasty," Int J Obes Relat Metab Disord, 22 (3). 222-226. Mar 1998.

[19] Cho, Y., Tokuhara, D., Morikawa, H., Kuwae, Y., Hayashi, E., Hirose, M., Hamazaki, T., Tanaka, A., Kawamura, T., Kawada, N. and Shintaku, H, "Transient Elastography-Based Liver Profiles in a Hospital-Based Pediatric Population in Japan," PLoS One, 10 (9) e0137239. Sep 2015.

[20] Hill, J.O. and Peters, J.C, "Biomarkers and functional foods for obesity and diabetes," Br J Nutr, 88 (suppl 2). S213-S218. Nov 2002.

[21] Astrup, A., Breum, L., Toubro, S., Hein, P. and Quaade, F, "The effect and safety of an ephedrine/caffeine compound compared to ephedrine, caffeine and placebo in obese subjects on an energy restricted diet. A double blind trial," Int J Obes Relat Metab Disord, 16 (4). 269-277. Apr 1992.

[22] Sjöström, L., Rissanen, A., Andersen, T., Boldrin, M., Golay, A. Koppeschaar, H.P. and Krempf, M, "Randomised placebocontrolled trial of orlistat for weight loss and prevention of weight regain in obese patients. European Multicentre Orlistat Study Group," Lancet, 352 (9123). 167-172. Jul 1998.

[23] Chaput, E., Saladin, R., Silvestre, M. and Edgar, A.D, "Fenofibrate and rosiglitazone lower serum triglycerides with opposing effects on body weight," Biochem Biophys Res Commun, 271 (2). 445-450. May 2000. 